\title{
Investigation on the Inert Gas-Assisted Laser Cutting Performances and Quality Using Supersonic Nozzles
}

\author{
Leonardo Orazi ${ }^{1,2, *(1)}$, Mohamed Darwish ${ }^{1}$ and Barbara Reggiani ${ }^{1}$ (1) \\ 1 DISMI-Department of Sciences and Methods for Engineering, University of Modena and Reggio Emilia, \\ Via Amendola 2, 42122 Reggio Emilia, Italy; mohamed.darwish@unimore.it (M.D.); \\ barbara.reggiani@unimore.it (B.R.) \\ 2 EN\&TECH-Interdepartmental Center, University of Modena and Reggio Emilia, Tecnopolo, \\ Piazzale Europa 1, 42124 Reggio Emilia, Italy \\ * Correspondence: leonardo.orazi@unimore.it; Tel.: +39-0522-522-607
}

Received: 28 October 2019; Accepted: 20 November 2019; Published: 25 November 2019

\begin{abstract}
In the present work, three different supersonic nozzles were designed and manufactured to operate at various stagnation pressures during laser cutting. Several cutting experiments were performed on stainless steel samples of various thicknesses $(2,4,6 \mathrm{~mm})$ using a fiber laser of $3 \mathrm{~kW}$ with a head adapted to fit with both the proposed supersonic nozzles and a commercial reference conical nozzle. The flow through these nozzles was numerically modeled and compared with the Schlieren visualization measurements. The mass flow rate, the Mach number, and the pressure distributions were selected in detail in order to analyze the dynamic characteristics of the exit jet and to comparatively assess the achieved cutting quality (roughness perpendicularity) and capability (maximum thickness, cutting speed). The numerical and the experimental results were found to be in high agreement in terms of the flow structure and mass flow rate. In addition, a significant reduction of the assistance gas consumption of up to $65 \%$ on average was achieved by using supersonic nozzles as opposed to conical ones, accompanied with the decrease of the operating pressure and the increase of the cutting speed.
\end{abstract}

Keywords: laser cutting; supersonic nozzles; stainless steel; roughness; Schlieren; energetic efficiency

\section{Introduction}

The gas-assisted process represents one of the most prominent methods for laser cutting of metallic parts, in which the molten phase is ejected with the help of an assistance gas flow. Both reactive gases, such as oxygen, and inert gases, such as nitrogen and argon, are commonly used. Oxidizing gas is mainly used for cutting ferrous alloys, such as mild steels, where the ferrous oxides are of low viscosity and the molten dross can be easily removed from the cut edge. In these cases, oxygen exothermically reacts with the metals, adding further energy and increasing the cutting efficiency, allowing operation at an inlet stagnation pressure lower than 5 bar. On the other hand, inert gas is usually selected to operate at a pressure of up to 30 bar for metals that are difficult to cut, such as stainless steels, aluminum, and titanium alloys, in order to avoid retention of molten oxides that are problematic to blow away from the cut kerf because their high viscosity [1,2].

The majority of the commercial nozzles are of a conical shape, given the low manufacturing costs. However, this benefit is counterbalanced by a very high gas-consumption rate which increases the operational cost, as well as by a small allowable stand-off distance $\left(X_{\text {off }}\right.$, nozzle tip-workpiece distance) that negatively affects the operating window in terms of material thickness. This small $\mathrm{X}_{\text {off }}$ condition is imposed due to the occurrence of undesirable aerodynamic phenomena, such as shock waves, through the exit jet flow of the conical nozzles operated at high pressures [3,4]. 
A comprehensive review of the dynamic characteristics of the exit jet from both conical and supersonic nozzles has been illustrated in $[5,6]$. The authors in [5] demonstrated that the exit jet from the supersonic nozzle is marked by a more uniform density distribution and by a higher momentum thrust if compared with the conical nozzle, resulting in the improvement of the cutting quality (roughness, perpendicularity) and capability (maximum thickness, cutting speed). The same authors in [6] experimentally proved that the cutting capability in terms of molten material removal and the allowable stand-off distance are enhanced by the use of a supersonic nozzle compared with a conical one.

Several cutting experiments have been conducted to illustrate the effect of nozzle types on the process parameters, namely cutting speed, laser power, stand-off distance, and workpiece thickness [7-14]. However, most of these experiments focused only on the cutting capability in terms of maximum achievable thickness rather than on the obtained cutting quality (the measured roughness). Cutting experiments on stainless steel samples using a diode laser were carried out, proving a faster speed for a thickness over $4 \mathrm{~mm}$ and a lower roughness with respect to what was achieved with a fiber laser [8].

In [9], the effect of the geometrical types of nozzles on the allowable operating tolerance of the stand-off distance was investigated on stainless steel samples of $60 \mathrm{~mm}$ thickness, demonstrating the greater operating tolerance for a Minimum Length Nozzle (MLN) and for commercial supersonic nozzles compared with conical subsonic ones.

Shin et al. [10] conducted several cutting experiments on stainless steel and carbon steel plates of various thicknesses of up to $100 \mathrm{~mm}$ by means of $6 \mathrm{~kW}$ fiber laser. As a result, an efficient cutting capability of $16.7 \mathrm{~mm}$ per $\mathrm{kW}$, accompanied with a high cutting speed, was achieved. In [11], the cutting performance was improved by preheating the workpiece before the operation, achieving the peak cutting speed with a step-like cutting speed increase technique.

Moreover, Tamura et al. [12] demonstrated that lasers can be efficiently utilized for cutting in nuclear decommissioning. They successfully executed cutting experiments for carbon steel and stainless steel plates of thicknesses of up to $300 \mathrm{~mm}$ using a $30 \mathrm{~kW}$ fiber laser.

Authors in [13] investigated the effects of various operating conditions in an oxygen gas-assisted laser cutting to obtain an optimum kerf width. Steel and mild steel samples with a thickness of 1 to $2 \mathrm{~mm}$ were processed using a laser power range of 50 to $170 \mathrm{~W}$, proving that a lower oxygen gas pressure ( 1 bar) is required to cut a mild steel sample of thickness $1 \mathrm{~mm}$, compared with a pressure of 4 bar to cut steel samples of the same thickness.

The behavior of the exit jet pattern within the cut kerf based on the jet flow-workpiece interaction has been numerically modeled and experimentally investigated in several works reported in literature in order to design different gas injection systems that help to avoid the drawbacks associated with the current conventional cutting nozzles [15-19].

Quintero et al. [15] analyzed and experimentally visualized the gas flow inside the cut kerf to illustrate the main factors controlling the aerodynamic interactions of the assistance gas with the workpiece. They demonstrated that the proper selection of the stand-off distance represents the essential factor in optimizing the molten material removal rate and in avoiding the formation of a recast layer on the cut edge.

Authors in $[16,17]$ numerically modeled the exit jet impinging on the cut kerf to analyze the effects of various laser cutting parameters (stand-off distance, exit Mach number, and workpiece thickness) on the formed shock structure. As a result, the direct interaction between the incident shock and the stand-off shock has been found to be mainly affected by the stand-off distance, while a large tolerance variation on this parameter is obtained at high Mach numbers. Similarly, Chen et al. [18,19] experimentally studied the achieved cutting quality and capability under various operating conditions and stand-off distances, confirming the dominant effects of the latter parameters.

Moreover, the exit jet from the supersonic nozzle in the free stream has been numerically studied to illustrate the behavior of the exit jet pattern under various operating conditions [20-22]. Zhang et al. [20] 
numerically modeled and visualized the exit jet patterns from two supersonic nozzles, a Minimum Length Nozzle (MLN) designed according to the Method of Characteristics (MOC) and an identical commercial supersonic nozzle with straight walls diverging by $5^{\circ}$. It was found that the MLN nozzle generates a uniform, bounded, and stable jet, extending for a longer distance (1.4 times) compared to the commercial one. Other authors in [21] investigated the gas-assisted laser cutting flow through a supersonic nozzle operated at high pressure under various operating conditions (desired-design, over-expansion, and under-expansion). The achieved results have been further investigated and accurately simulated by means of an efficient model developed by the authors of the current work [22]; the same model was used to simulate the exit jet behavior from the nozzles proposed in this article under various operating stagnation pressures [23].

Even in view of the existing literatures on the subject, most of the reported experimental work has been focused mainly on the achieved cutting capability (cutting thickness) rather than on the cutting quality (the measured roughness and perpendicularity of the cut edge). In addition, to the best of the author's knowledge, no previous works reported indications of the effective gas consumption in laser cutting.

\section{Materials and Methods}

In this framework, the present work was aimed at investigating the effects of nozzle types and operating conditions on the achieved cutting quality and capability. Three different supersonic nozzles were designed based on the gas dynamics theory and fabricated by means of Wire Electrical Discharge Machining (WEDM). The numerically simulated flow through these supersonic nozzles was compared with the experimental observation obtained by Schlieren visualization.

Several laser cutting experiments were conducted by using an nLIGHT alta ${ }^{\mathrm{TM}}$ Fiber Laser (nLIGHT Inc., Vancouver, WA, USA) emitting a maximum power of $3 \mathrm{~kW}$ at $1.08 \mu \mathrm{m}$ wavelength on stainless steel samples made by AISI 316 of various thicknesses $(2,4$, and $6 \mathrm{~mm})$. The performances of the supersonic nozzles in terms of cutting speed and edge quality were evaluated, and the outcomes were compared with those of a commercial conical nozzle. Moreover, the cutting edge perpendicularity-which represents one of the critical quality aspects-was calculated for each supersonic nozzle and compared with that of the reference conical nozzle. The comparative analysis of the achieved cutting quality was conducted operating under the exact-design condition specified for each supersonic nozzle, and under a fixed stagnation pressure of $20 \mathrm{bar}$, indicated as the optimal condition for the reference conical nozzle by prior experiments. This choice can be supported considering that the gas-assisted laser cutting flow shows optimum dynamic characteristics under the exact-design condition-calculated for each nozzle - so that optimal cutting quality can be obtained under these different operating settings [22,23].

Furthermore, the gas consumption efficiency was comparatively assessed for each supersonic nozzle with respect to the reference conical nozzle.

\section{Supersonic Nozzle Optimal Design}

The supersonic nozzle's design was based on the quasi 1-D gas dynamic theory stating that the desired-design condition is achieved when the inlet operating pressure is exactly equal to the computed one based on the nozzle area ratio. The flow is assumed to be irrotational, isentropic, compressible, and supersonic, and the exit Mach number $M_{e}$ is defined by the isentropic relationship between Mach number and nozzle area ratio as in the following Equation (1) [3,4,24-26]:

$$
\frac{A_{e}}{A^{*}}=\frac{1}{M_{e}}\left(\frac{1+\frac{\gamma-1}{2} M_{e}^{2}}{1+\frac{\gamma-1}{2}}\right)^{\frac{\gamma+1}{2(\gamma-1)}}
$$

where $\gamma=1.4$ is the specific heat ratio, $A_{e}$ the exit area, and $A^{*}$ the throat area. 
Once the Mach number is determined, the ratio of the inlet stagnation pressure $P_{o}$ to the exit pressure $P_{e}$ is defined as in Equation (2):

$$
\frac{P_{o}}{P_{e}}=\left(1+\frac{\gamma-1}{2} M_{e}^{2}\right)^{\frac{\gamma}{\gamma-1}}
$$

Under the desired-design condition, the potential energy of the stagnation pressure is efficiently converted to kinetic energy, and the gas-assisted laser cutting flow is uniformly expanded with an exit pressure $\left(P_{e}\right)$ equal to the ambient one $(1 \mathrm{~atm})$. Based on the aforementioned criteria, three different supersonic nozzles, labeled SNA, SNB, and SNC, have been designed to operate under three various conditions: $14.25,7.45$, and 4.47 bars, respectively. The outer dimensions of these supersonic nozzles were taken, and coincided with those of a commercial conical one used as reference and labelled CN0. The dimensions and the operation conditions of the nozzles are summarized in Table 1 and shown in Figure 1. Small differences between the designed and the actual convergent lengths arose from WEDM manufacturing constraints, resulting in $0.5 \mathrm{~mm}$ of straight length of the throat section, as shown in Figure 1a.

Table 1. Supersonic nozzle dimensions and exact operating conditions.

\begin{tabular}{cccccc}
\hline Parameters & Units & SNA & SNB & SNC & CN0 \\
\hline Inlet Diameter $\left(D_{i}\right)$ & $\mathrm{mm}$ & 8.0 & 8.0 & 8.0 & 8.0 \\
\hline Throat Diameter $\left(D^{*}\right)$ & $\mathrm{mm}$ & 1.5 & 1.8 & 2.0 & - \\
\hline Exit Diameter $\left(D_{e}\right)$ & $\mathrm{mm}$ & 2.3 & 2.3 & 2.3 & 2.3 \\
\hline Nozzle length $(L)$ & $\mathrm{mm}$ & 15.5 & 15.5 & 15.5 & 15.5 \\
\hline Designed Convergent length $\left(L_{C 1}\right)$ & $\mathrm{mm}$ & 14.0 & 12.5 & 14.0 & 15.5 \\
\hline Actual Convergent length $\left(L_{C}\right)$ & $\mathrm{mm}$ & 13.5 & 12.0 & 13.5 & 15.5 \\
\hline Divergent length $\left(L_{D}\right)$ & $\mathrm{mm}$ & 1.5 & 3.0 & 1.5 & 0.0 \\
\hline Entrance (inlet) angle $\left(\theta_{I}\right)$ & degree & $27.07^{\circ}$ & $28.97^{\circ}$ & $24.89^{\circ}$ & $20.85^{\circ}$ \\
\hline Exit (Divergence) angle $\left(\theta_{e}\right)$ & degree & $29.86^{\circ}$ & $9.52^{\circ}$ & $9.90^{\circ}$ & - \\
\hline Exact Inlet Stagnation Pressure $\left(P_{o}\right)$ & bar & 14.3 & 7.5 & 4.5 & 20.0 \\
\hline Exact Exit Pressure $\left(P_{e}\right)$ & bar & & & 1.01325 & \\
\hline Exact Exit Mach Number $\left(M_{e}\right)$ & & 2.37 & 1.96 & 1.63 & - \\
\hline
\end{tabular}

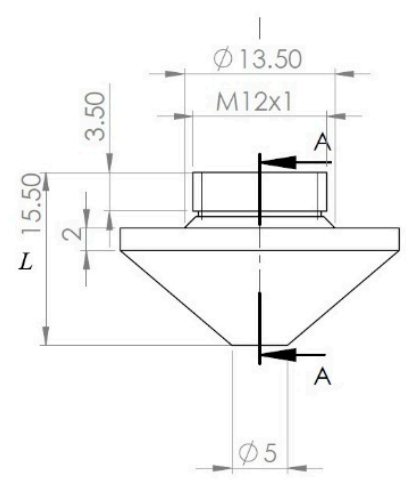

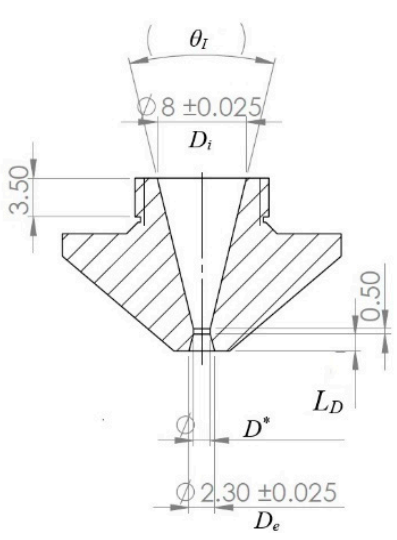

(a)

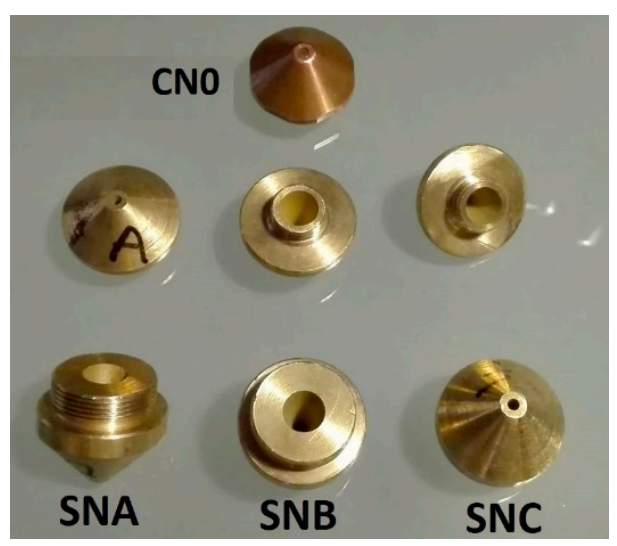

(b)

Figure 1. Supersonic nozzle outlines: (a) Supersonic nozzle dimensions; (b) Manufactured nozzles using WEDM. 
Like most commercial nozzles, the nozzles were designed with straight walls due to their easy manufacturing, while the exit divergence angle $\left(\theta_{e}\right)$ was initially set equal to $30^{\circ}$ for SNA and $10^{\circ}$ for both SNB and SNC [27]. Then, the throat diameter $\left(D^{*}\right)$ and nozzle inlet angle $\left(\theta_{I}\right)$ were calculated from Equations (3) and (4), respectively:

$$
\begin{gathered}
D^{*}=D_{e}-2 * L_{D} * \tan \left(\frac{\theta_{e}}{2}\right) \\
\tan \left(\frac{\theta_{I}}{2}\right)=\left(\frac{D_{i}-D^{*}}{2 * L_{C}}\right)
\end{gathered}
$$

After the design stage, the proposed nozzle design was checked to assess the manufacturability of the angles and lengths of both the two internal cones (convergent and divergent) by a 4-axis WEDM machine. This check was done based on the design criteria condition given in Equation (5):

$$
\left(\frac{\theta_{I}}{2}\right)<\tan ^{-1}\left(\frac{D^{*}}{2 * L_{D}}\right)
$$

\section{Numerical Model and Simulation Results}

The gas-assisted laser cutting flow was simulated using the model developed in [22]. The compressible flow through the proposed nozzles (SNA, SNB, and SNC) is governed by the Reynolds-Averaged Navier-Stokes (RANS) equations, where all of the unsteadiness is averaged out and regarded as part of the turbulence, together with two additional turbulence equations $(k-\varepsilon)$. The sonicFoam solver was applied, as it is more adequate for solving the intended governing equations considering the turbulence viscosity effect [28-30]. The fluid was modeled as air, given the fact that nitrogen cutting gas is quite similar. Two hexahedral blocks with cell sizes of $0.05 \mathrm{~mm}$ were used to generate both the nozzle geometry and the buffer section, which was added to simulate the exit jet. Moreover, the nozzle geometry was efficiently modeled by utilizing the two-dimensional axi-symmetric feature, and by modelling a wedge cross section with an angular extension of $2.5^{\circ}$ instead of the whole nozzle geometry. Finally, the same simulation control parameters, discretization schemes, solution algorithm, and initial boundary conditions reported in [22] were applied, except that a no-slip boundary condition was set up for the velocity at the nozzle wall.

The numerical results, Mach number, and pressure distributions, under the desired-design condition for the nozzles SNA, SNB, and SNC and under the operating condition for CN0, are shown in Figures 2 and 3, respectively.

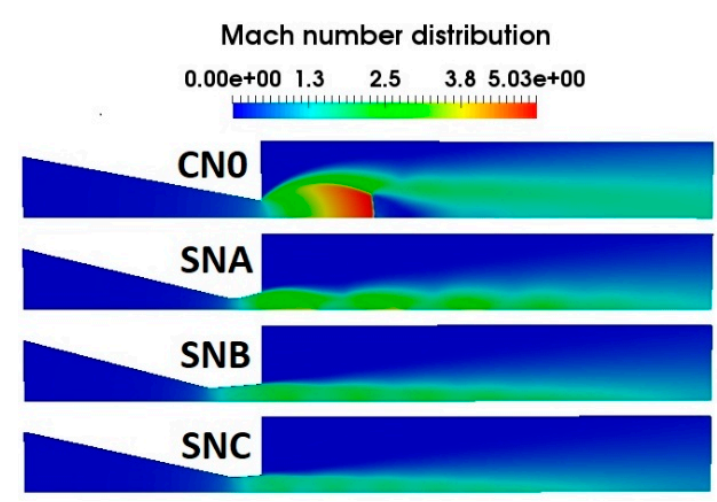

(a)

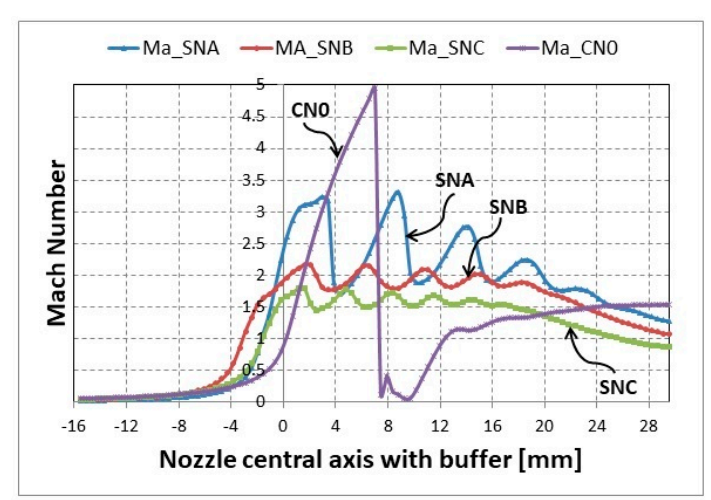

(b)

Figure 2. Mach number distribution of the different nozzles under the desired-design condition: (a) Mach number distribution map; (b) Mach number distribution along the central axes. 


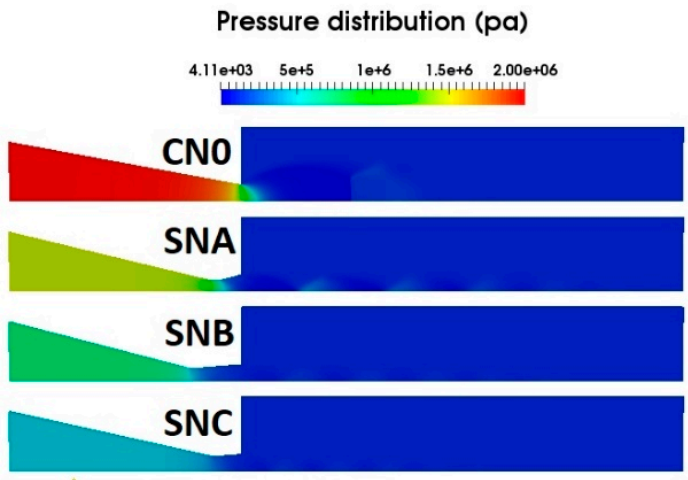

(a)

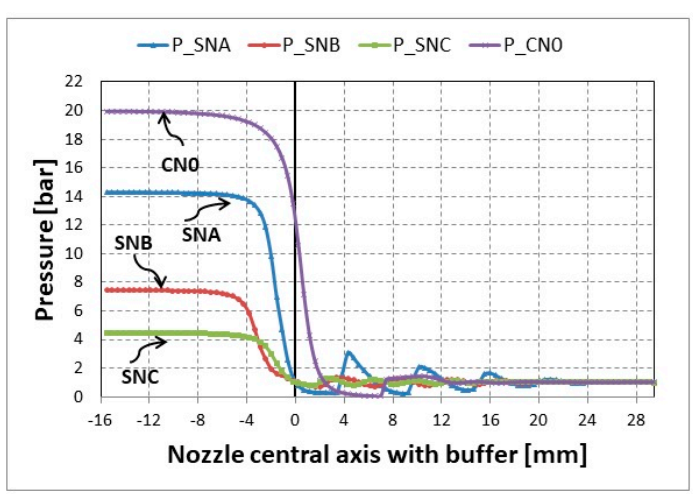

(b)

Figure 3. Pressure distribution of the different nozzles under the desired-design condition: (a) Pressure distribution map; (b) Pressure distribution along the central axes.

As can be seen, the numerical results demonstrate that, under the desired-design condition, the gas-assisted laser cutting flow uniformly expands through the proposed supersonic nozzles without the occurrence of any shock waves in the divergence sections. The fluctuations in the jet after the tip can be explained by the fact that the proposed nozzles are fabricated with straight walls, and this partially fulfills the quasi 1-D assumptions. Moreover, the higher flow fluctuations from nozzle SNA arise from its high exit divergence angle. However, the exit jet from the conical nozzle is subjected to the formation of strong oblique and normal shock waves, and the strength of these waves is increased with the increase of the inlet stagnation pressure $\left(P_{o}\right)$, resulting in the non-uniformity of the exit jet density distribution and, eventually, in the reduction of the expected cutting quality and capability.

In Table 2, the numerical results along the central axes of the conical and supersonic nozzles under the desired-design condition are reported together with the mass flow rate and the percentage difference with respect to the theoretical desired-design values computed by the quasi 1-D gas dynamics theory.

Table 2. Simulations results of the supersonic nozzles under desired-design condition and the reference conical nozzle.

\begin{tabular}{cccccc}
\hline Parameters & Units & SNA & SNB & SNC & CN0 \\
\hline Exit Mach No. $M_{e}$ simulated & - & 2.25 & 1.90 & 1.60 & 0.94 \\
\hline Quasi 1-D exit Mach no. $M_{e}$ & - & 2.37 & 1.96 & 1.63 & - \\
\hline $\mathrm{M}_{e}$ difference & - & $-6.3 \%$ & $-3.1 \%$ & $-1.6 \%$ & - \\
\hline Exit Pressure simulated $P_{e, s i m}$ & $(\mathrm{~Pa})$ & 114,650 & 108,100 & 101,500 & $1,349,400$ \\
\hline Quasi 1-D exit Pressure $P_{e, t h}$ & $(\mathrm{~Pa})$ & & 101325 & & - \\
\hline$\Delta P_{e}=P_{e, s i m}-P_{e, t h}$ & - & $+13 \%$ & $+7 \%$ & $+1 \%$ & - \\
\hline Exit Velocity $U_{e}$ at nozzle tip & $\left(\mathrm{m} \cdot \mathrm{s}^{-1}\right)$ & 556 & 495 & 448 & 311 \\
\hline Mass flow rate simulated & $\left(\mathrm{g} \cdot \mathrm{s}^{-1}\right)$ & 6.05 & 4.98 & 3.95 & 14.30 \\
\hline
\end{tabular}

Table 2 demonstrates that the nozzle SNA has the highest percentage error between the exactdesign and the numerically predicted values, since it was designed with the largest divergence angle $\left(\theta_{e}=30^{\circ}\right)$ compared to the other nozzles. Moreover, Table 2 clearly indicates that a reduction of the gas consumption rate of $55 \%, 63 \%$, and $70.5 \%$ for nozzles SNA, SNB, and SNC, respectively, is expected compared to the reference nozzle $\mathrm{CN} 0$, marked by a mass flow rate of $14.3 \mathrm{~g} / \mathrm{s}$. 


\section{Experimental Set-Up}

The Schlieren technique is widely applied to visualize numerous engineering phenomena. It is recognized that the optical flow visualization is the most suitable method to observe the shock structures in the compressible flow. Authors in [31] qualitatively investigated and experimentally visualized the shock wave diffraction phenomena around two splitters with spike-shaped structures for different Mach numbers. Schlieren photography was used to obtain an insight into the sequential diffraction processes that take place in different planes and as a result, a complete description of the main flow features was successfully provided. In addition, Schlieren technique represents one of the simplest optical visualizing procedures, especially convenient for observing the intermittent shock structures in the gas jet. For these reasons, the simulation results were compared with experimental characterizations based on the use of the Schlieren method. The scheme of the test facility is shown in Figure 4; it consisted of a nitrogen $\left(N_{2}\right)$ compressor adapted to provide compressed $N_{2}$ up to 25 bar. This compressor was connected to a valve in order to regulate the supply of $N_{2}$ into the tested nozzles, to a flow meter to measure the volume flow rate $(\mathrm{L} / \mathrm{min})$, and to a pressure sensor to adjust the required pressure. Then, this assembly was connected to the nozzle through the nozzle holder. Furthermore, an optical system was provided to capture the images aimed at investigating the dynamic behavior of the gas flow from the nozzle. This induced a change in gas pressure and temperature and caused a change of the refractive index, which deviated the divergent light beam from the pinpoint light source going through the test area. The non-deviated light beam was filtered by a knife edge placed at a focal point. The deviated light beam was projected onto the image plane. With the best alignment, the undisturbed light beam should form a spot at the knife edge, whose dimensions match the diameter of the pinhole used in the spatial filter. A CMOS camera captured the dynamic behavior of the gas flow from the nozzle.

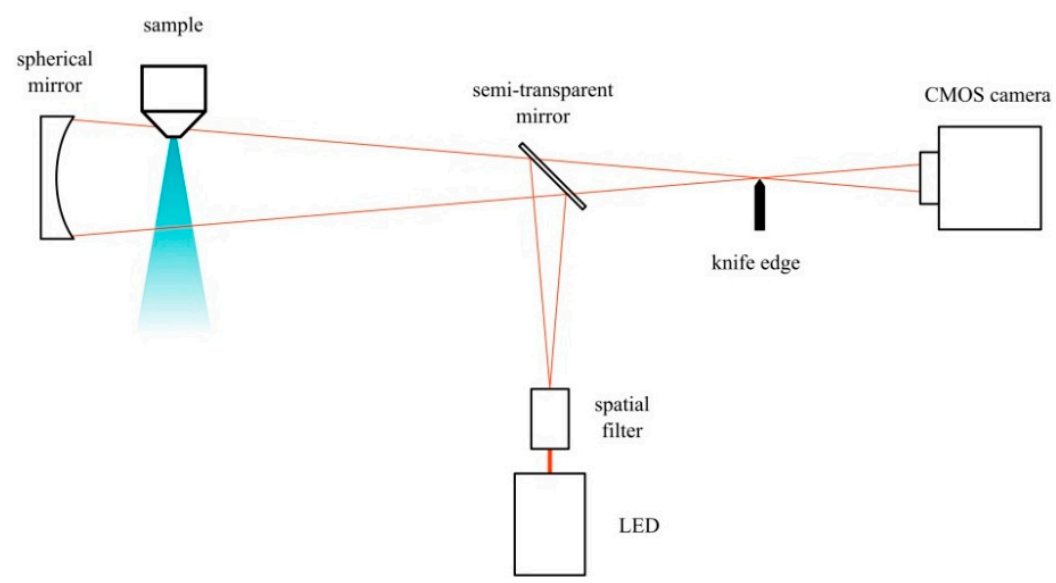

Figure 4. Schematic diagram of the Schlieren setup.

The whole gas flow was imaged using a spherical mirror with focal length of $2000 \mathrm{~mm}$ and diameter of $300 \mathrm{~mm}$. Furthermore, for detailed images including the intermittent shock structures (Mach disks), a smaller spherical mirror with focal length of $650 \mathrm{~mm}$ and diameter of $63 \mathrm{~mm}$ was used [23,31-33].

During the Schlieren measurements, the mass flow rate was experimentally estimated by the measured volume flow and the knowledge of the gas density. The resulting mass flow rates were calculated for the nitrogen-assisted gas at the sea level condition $\left(15^{\circ} \mathrm{C}, 1013.25 \mathrm{hPa}\right)$ and a density $(\rho)$ equal to $1.185 \mathrm{~kg} / \mathrm{s}^{3}$, as summarized in Table 3 . 
Table 3. Schlieren operating conditions, measured volume flow rates, and calculated mass flow rates.

\begin{tabular}{cccc}
\hline Nozzle & Operating Pressure & Volume flow rates $(l / m)$ & Mass flow rates $(\mathrm{g} / \mathrm{s})$ \\
\hline \multirow{3}{*}{ SNA } & Under-expansion: 20 bar & 458 & 9.05 \\
& Desired-design: 14.25 bar & 327 & 6.46 \\
& Over-expansion: 8 bar & 175 & 3.46 \\
\hline \multirow{3}{*}{ SNB } & Under-expansion: $10 \mathrm{bar}$ & 375 & 7.41 \\
& Desired-design: 7.4 bar & 274 & 5.42 \\
& Over-expansion: 4 bar & 171 & 3.38 \\
\hline \multirow{3}{*}{ SNC } & Under-expansion: 8 bar & 370 & 7.31 \\
& Desired-design: 4.47 bar & 218 & 4.31 \\
& Over-expansion: 3 bar & 162 & 3.20 \\
\hline \multirow{6}{*}{ CN0 } & 20 bar & 852 & 16.84 \\
& 14.25 bar & 677 & 13.38 \\
& 7.4 bar & 300 & 5.93 \\
& 4.47 bar & 258 & 5.10 \\
\hline
\end{tabular}

Laser cutting tests were then conducted by the laser system shown in Figure 5. The workpiece samples were made by AISI 316 stainless steel plates of different thicknesses: 2, 4, and $6 \mathrm{~mm}$. All the experiments were conducted operating at $3 \mathrm{~kW}$ and using a $100 \mu \mathrm{m}$ delivery fiber core diameter; the laser beam was collimated using a $100 \mathrm{~mm}$ lens and focused using a $150 \mathrm{~mm}$ focal lens.

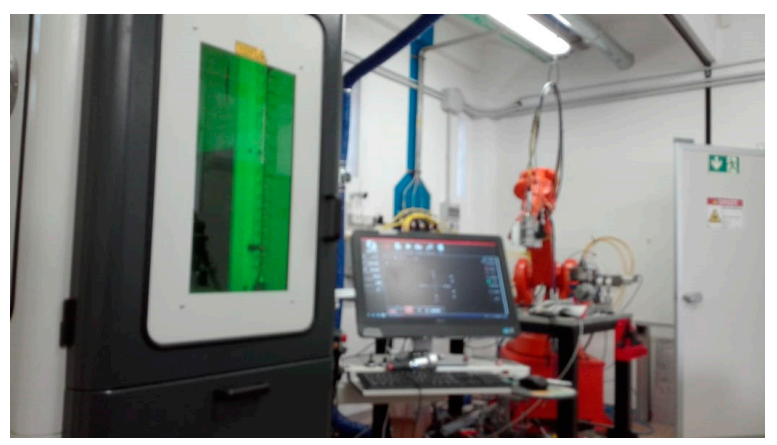

(a)

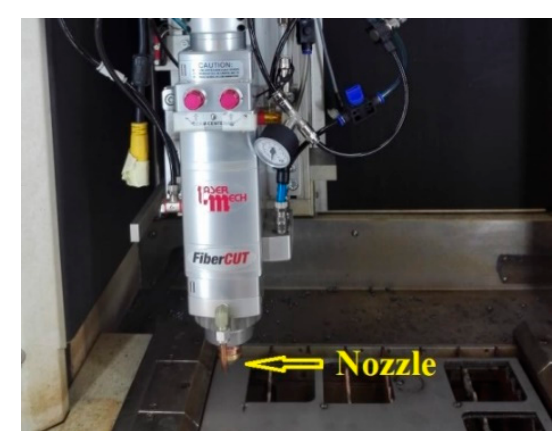

(b)

Figure 5. Laser cutting system: (a) Working cabinet and control panel; (b) Laser head.

A laser cutting head (Figure 5b), fitted with either the reference conical or the developed supersonic nozzles, was used, and nitrogen was introduced coaxially with the focused beam via these nozzles regulating the gas pressure between 4.5 and 20 bar.

During the laser cutting experiments, the process parameters in the case of the reference conical nozzle were chosen by following the best practices developed under prior experiments. Thus, this nozzle was operated at a fixed stagnation pressure of 20 bar, fixed focal position equal to $-4 \mathrm{~mm}$, and cutting speed of 4.5 and $2 \mathrm{~m} / \mathrm{min}$ for the specimens of thickness 2 and $4 \mathrm{~mm}$, respectively. In addition, the entire cutting experiments were conducted at a low stand-off distance equal to $0.5 \mathrm{~mm}$ to avoid the deterioration of the dynamic characteristics and momentum thrust after the formed strong normal shock wave associated with this high operating pressure.

On other hand, the process parameters for the supersonic nozzles were differently specified. The operating stagnation pressure was calculated for each nozzle according to the exact-design condition, while the stand-off distance was fixed at the same reference value of $0.5 \mathrm{~mm}$, although the exit jet from the supersonic nozzle is characterized by higher dynamic characteristics compared to those of the conical one, allowing safer operation at a longer stand-off distance. Finally, the focal position and cutting speed were selected based on several experimental trials, and the reported results were accompanied with the optimal achieved cutting quality. 
The cutting-edge quality was investigated by using a stylus profilometer Hommelwerke TESTER T500 (Hommelwerke GmbH, Villingen-Schwenningen, Germany), and the measured values analyzed via the TURBO DATAWIN PC software (Hommelwerke $\mathrm{GmbH}$, Villingen-Schwenningen, Germany). The traverse length $\left(l_{t}\right)$, which represents the evaluation length, was set to $15 \mathrm{~mm}$, while the cut-off length $\left(l_{c}\right)$ was selected equal to $2.5 \mathrm{~mm}$. The roughness parameters, average roughness $\left(\mathrm{R}_{\mathrm{a}}\right)$ and average maximum height of the profile $\left(R_{z}\right)$, were measured on each cutting edge at least five times for each sample edge, calculating the mean value and the absolute deviation of the measured roughness values. These measurements of roughness parameters were conducted at the centerline along the cut thickness of the cut edge to unify the comparative assessment of the achieved cutting quality from all nozzles. Moreover, the cutting edge perpendicularity was evaluated with the help of a structured light 3D scanner GOM ATOS Core 2000 (GOM GmbH, Braunschweig, Germany).

\section{Experimental Results and Discussion}

\subsection{Flow Visualization and Mass Flow Rate Measurements}

The exit jet patterns from the supersonic nozzles were experimentally visualized under various operating conditions (desired-design, over-expansion, and under-expansion) while CN0 was visualized under both the sonic stagnation pressure of 1.89 bar and under the same operating pressure conditions of SNA, SNB, and SNC, as tabulated in Table 3.

The exit jet patterns from the nozzles SNA, SNB, and SNC, operated under the desired-design condition, and, for comparison, from the nozzle $\mathrm{CNO}$, are shown in Figure 6a-c. Then, the intermittent shock structures (i.e., Mach disks), through the exit jet of the supersonic nozzles, was magnified using the smaller spherical mirror, as depicted in Figure 6d.

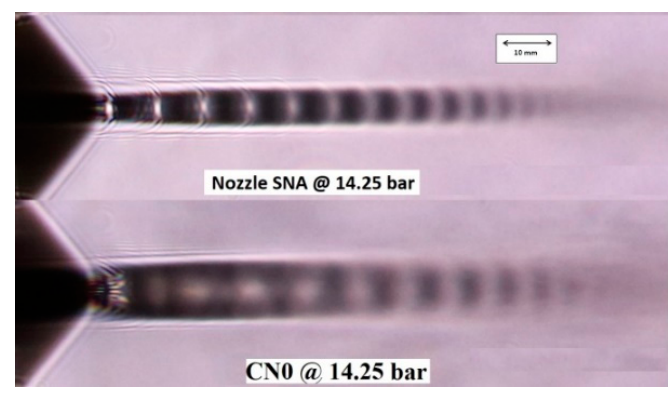

(a)

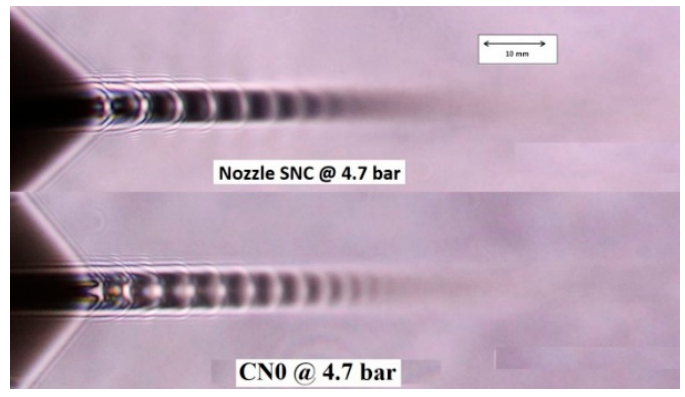

(c)

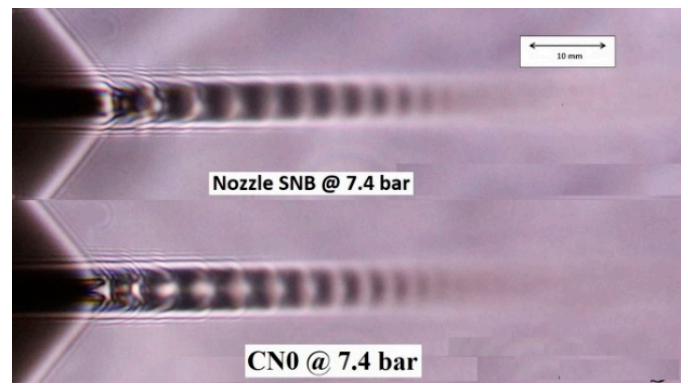

(b)

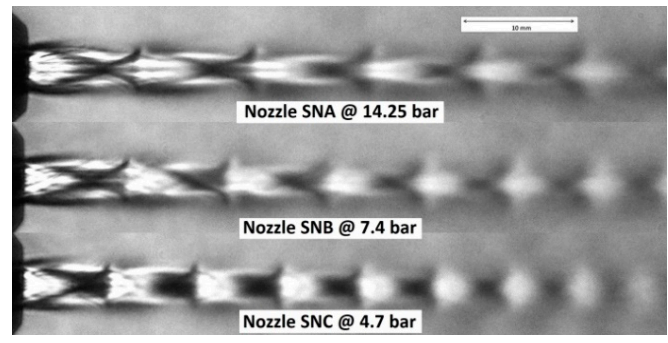

(d)

Figure 6. Schlieren visualizations: (a) SNA vs. CN0 at (@) 14.25 bar; (b) SNB vs. CN0 @ 7.4 bar; (c) SNC vs. CN0 @ 4.7 bar; (d) The exit jet patterns from SNA, SNB, and SNC at high magnification.

Figure 6 clearly demonstrates that the exit jet from the proposed supersonic nozzles, under the exact operating condition, is marked by high uniformity and has a more dense flow distribution and tidy boundary compared to the flow pattern obtained by the reference conical nozzle; hence, 
the expected cutting quality and capability are enhanced. Moreover, the length of the effective exit jet $\left(L_{e f f}\right)$, which is measured from the nozzle tip up to the point where the flow starts to diverge, is increased with the inlet stagnation pressure. The nozzle SNA thus showed the longest $L_{\text {eff }}$ compared to the other two nozzles and the reference conical one.

Figure $6 \mathrm{a}-\mathrm{c}$ prove that the exit jet from $\mathrm{CN} 0$ is strongly under-expanded, since the operating pressure exceeds the sonic stagnation pressure (1.89 bar). Thus, the flow density distribution and the momentum thrust of the exit jet are degraded, resulting in an increase of the expected gas consumption compared to the proposed supersonic nozzles.

Although for the conical nozzle, the effective exit jet length $\left(L_{e f f}\right)$ is increased with the increase of the operating stagnation pressure, the width of the exit jet is also increased, since the flow becomes strongly under-expanded, causing the deterioration of the flow density distribution [23].

Finally, Figure 2, Figure 3, and Figure 6 highlight that the numerically predicted results and visualization measurements are in high agreement in terms of the shapes of periodic waves.

The volume flow rates through all of the nozzles were measured by the flow meter under the various operating conditions, representing direct indicators of the gas consumption. For CN0, Table 3 shows that the wastage of gas-assisted laser is significantly increased with the increase of the inlet stagnation pressure.

The numerically predicted mass flow rates and the measured values through Schlieren experiments, for both the supersonic nozzles operated at the desired-design conditions and for the reference conical nozzle operated at 20 bar, are presented in Figure 7. This comparison allows the comparative assessment of the achieved reduction by the supersonic nozzles with respect to $\mathrm{CN} 0$ and the evaluation of the reliability of the numerical model. In detail, Figure 7 demonstrates that the conical nozzle $\mathrm{CN} 0$, operated at 20 bar, has the highest mass flow rate compared to the other supersonic nozzles, specifically 2.6, 3.1, and 3.9 times higher than those of SNA, SNB, and SNC, respectively. Moreover, the experimentally measured mass flow rates are quite close to the predicted numerical values, which are only slightly higher, with a percentage error ranging between $6.4 \%$ and $13.5 \%$, thus suggesting a very good agreement.

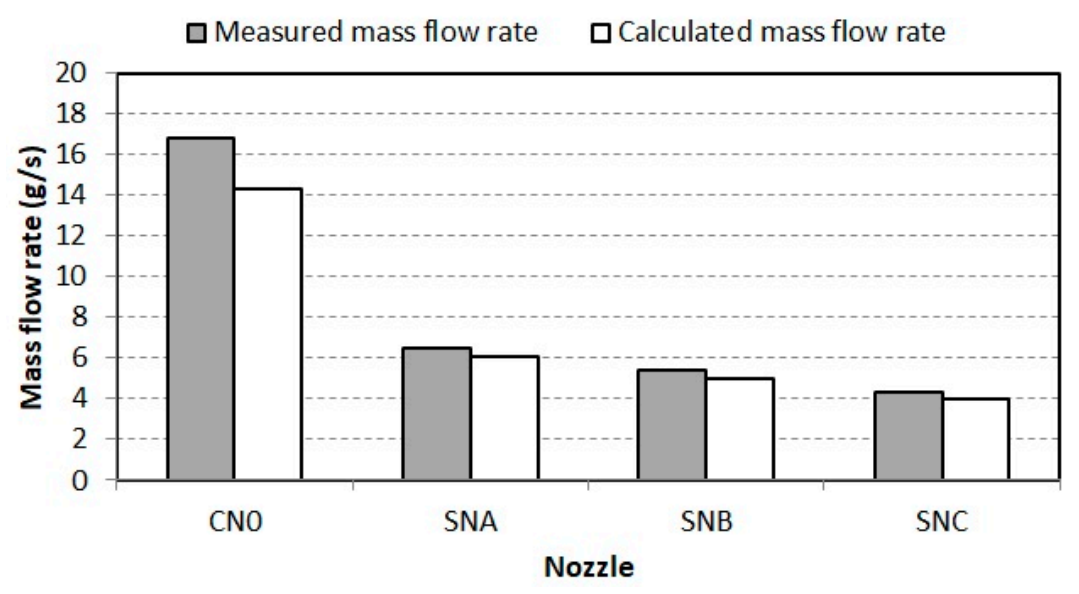

Figure 7. Measured and calculated mass flow rate.

\subsection{Cutting Experiment Results}

During the experiments, the laser power and the stand-off distance were fixed at $3 \mathrm{~kW}$ and $0.5 \mathrm{~mm}$, while the cutting speed was varied between 2 and $8 \mathrm{~m} / \mathrm{min}$ and the focal position between $-3.5 \mathrm{~mm}$ and $-6.5 \mathrm{~mm}$ below the workpiece surface. The workpiece was placed on a CNC $x-y$ table below the stationary laser beam, as shown in Figure 5. The cutting performance was evaluated in terms of the amount of dross build up (i.e., burrs) and the cut edge quality by measuring the surface roughness of the cut samples. Moreover, the cutting edge perpendicularity was measured for all the samples to further assess the achieved cutting quality. 
As previously reported, the cutting experiments were conducted on three different thicknesses $(2,4$, and $6 \mathrm{~mm})$ and the results were reported in Figure 8, Figure 9, and Figure 10 for the 2, 4, and $6 \mathrm{~mm}$ thicknesses, respectively. The arrows in these figures point to the bottom of the cut kerf since all are in the same direction, except the $4 \mathrm{~mm}$ sample cut by SNB which is reported in the reverse direction to clearly show the formed burrs at the bottom of the cut kerf. The measured roughness parameters and the measured cutting edge perpendicularity are summarized in Table 4 . It must be outlined that the cutting of the $6 \mathrm{~mm}$ thickness samples was not feasible (incomplete cutting state) with the reference conical nozzle CN0, since the specified operating condition is not matched with this plate thickness under the specified cutting speed.

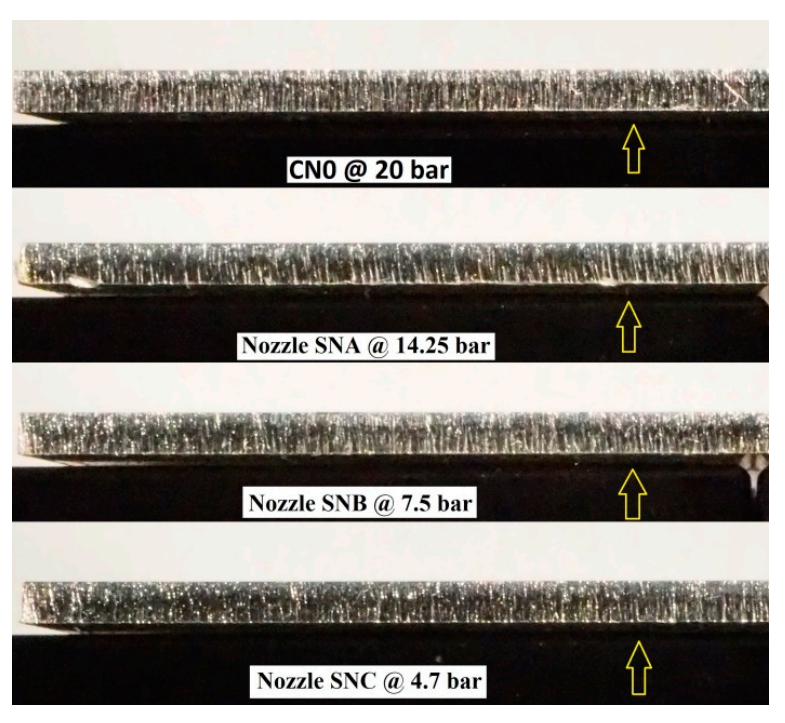

(a)

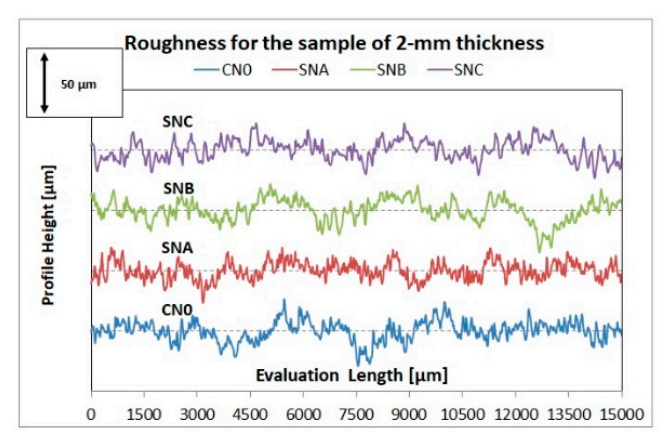

(b)

Figure 8. Cutting experiments: (a) $2 \mathrm{~mm}$ thickness samples (arrows refer to the bottom of the cut kerf); (b) Measured roughness.

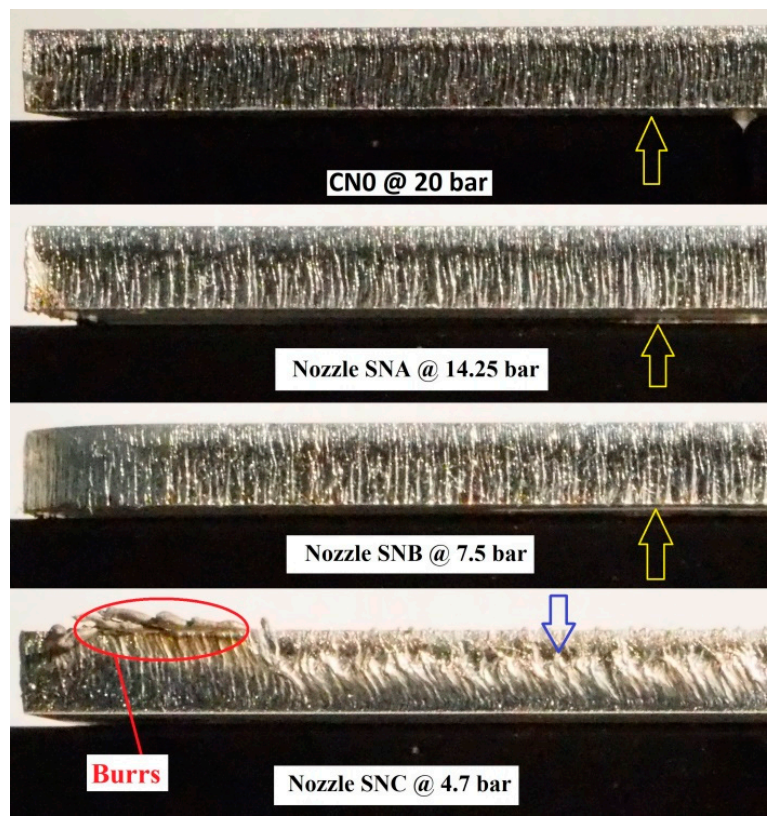

(a)

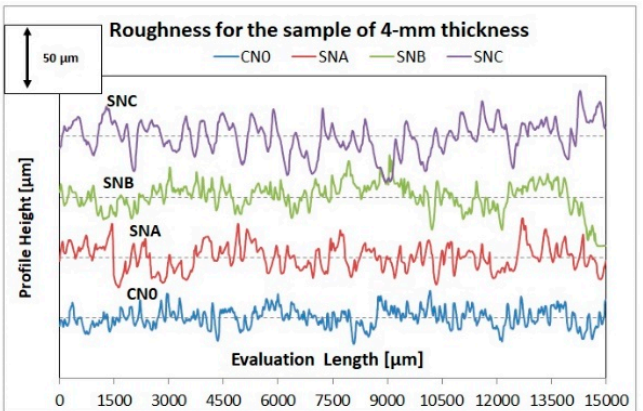

(b)

Figure 9. Cutting experiments: (a) 4 mm thickness samples (arrows refer to the bottom of the cut kerf); (b) Measured roughness. 


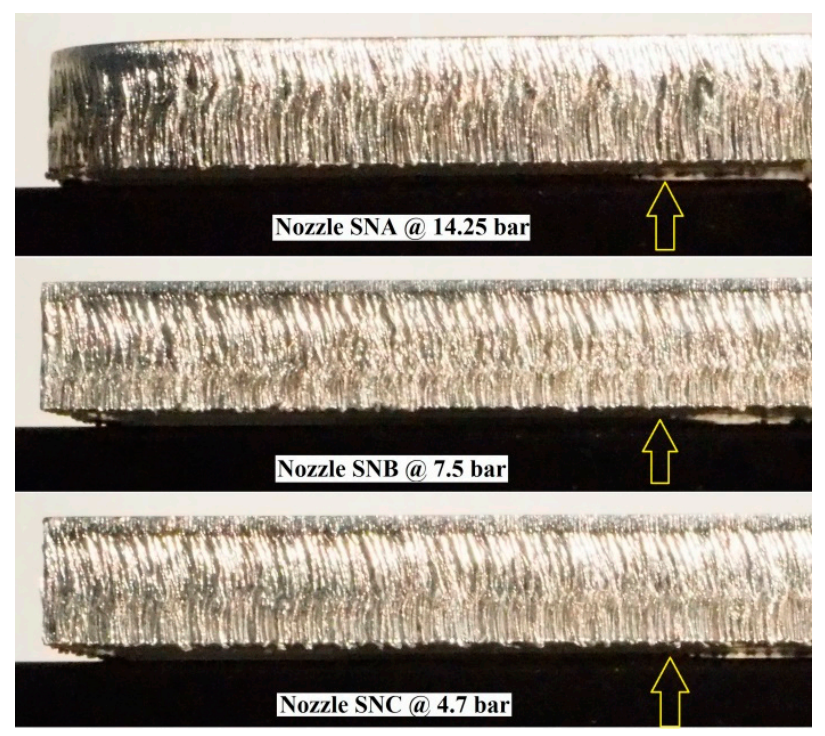

(a)

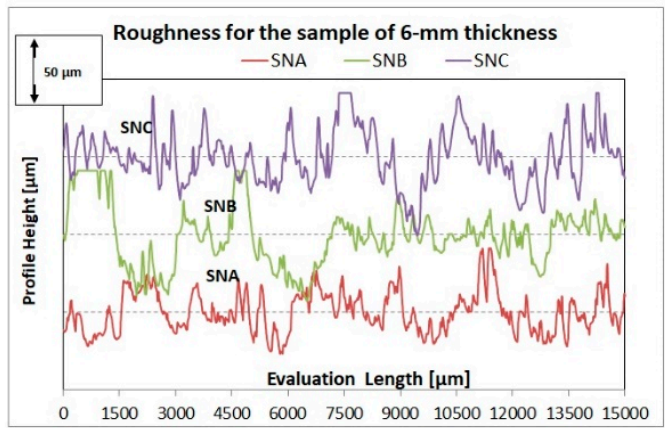

(b)

Figure 10. Cutting experiments: (a) $6 \mathrm{~mm}$ thickness samples (arrows refer to the bottom of the cut kerf); (b) Measured roughness.

Table 4. Cutting experiments conditions and measured roughness parameters.

\begin{tabular}{|c|c|c|c|c|c|c|c|}
\hline \multirow{2}{*}{$\begin{array}{l}\text { Sample } \\
\text { Thickness }\end{array}$} & \multirow{2}{*}{$\begin{array}{l}\text { Used } \\
\text { Nozzle }\end{array}$} & \multicolumn{2}{|c|}{ Operating Conditions } & \multirow{2}{*}{$\begin{array}{c}\text { Focal } \\
\text { Position } \\
{[\mathrm{mm}]}\end{array}$} & \multicolumn{2}{|c|}{$\begin{array}{l}\text { Roughness Parameters } \\
{[\mu \mathrm{m}]}\end{array}$} & \multirow{2}{*}{$\begin{array}{c}\text { Cutting Edge } \\
\text { Perpendicularity } \\
{\left[^{\circ}\right]}\end{array}$} \\
\hline & & $\mathbf{P}$ [bar] & $\mathrm{U}[\mathrm{m} / \mathrm{min}]$ & & $\mathbf{R}_{\mathrm{a}}$ & $\mathbf{R}_{\mathrm{z}}$ & \\
\hline \multirow{4}{*}{$2 \mathrm{~mm}$} & CNO & 20.00 & 4.5 & -4.0 & $5.04 \pm 0.06$ & $31.81 \pm 0.16$ & $1.10 \pm 0.09$ \\
\hline & SNA & 14.25 & 8.0 & -6.5 & $5.25 \pm 0.16$ & $31.44 \pm 0.15$ & $3.60 \pm 0.98$ \\
\hline & SNB & 7.50 & 6.0 & -3.5 & $4.98 \pm 0.08$ & $28.93 \pm 0.24$ & $0.40 \pm 0.80$ \\
\hline & SNC & 4.70 & 4.5 & -3.5 & $4.82 \pm 0.02$ & $29.04 \pm 0.31$ & $2.70 \pm 0.70$ \\
\hline \multirow{4}{*}{$4 \mathrm{~mm}$} & CNO & 20.00 & 2.0 & -4.0 & $5.45 \pm 0.04$ & $37.72 \pm 0.31$ & $1.60 \pm 0.13$ \\
\hline & SNA & 14.25 & 4.0 & -6.5 & $7.47 \pm 0.17$ & $40.25 \pm 0.61$ & $1.20 \pm 1.18$ \\
\hline & SNB & 7.50 & 3.5 & -6.5 & $5.53 \pm 0.11$ & $37.44 \pm 0.75$ & $0.60 \pm 0.22$ \\
\hline & SNC & 4.70 & 5.0 & -5.0 & $9.37 \pm 0.39$ & $51.58 \pm 0.81$ & $3.10 \pm 0.84$ \\
\hline \multirow{3}{*}{$6 \mathrm{~mm}$} & SNA & 14.25 & 2.5 & -4.0 & $8.65 \pm 0.11$ & $42.05 \pm 0.15$ & $1.40 \pm 0.18$ \\
\hline & SNB & 7.50 & 2.0 & -4.0 & $8.59 \pm 0.33$ & $40.07 \pm 1.57$ & $1.40 \pm 0.15$ \\
\hline & SNC & 4.70 & 2.0 & -4.0 & $11.46 \pm 1.40$ & $60.91 \pm 3.32$ & $1.30 \pm 0.61$ \\
\hline
\end{tabular}

The cutting and the gas consumption efficiencies were calculated for each sample according to Equations (6) and (7), respectively.

$$
\begin{aligned}
\xi & =\left(\frac{t v}{p}\right) \\
\eta & =\left(\frac{m}{t v}\right)
\end{aligned}
$$

where $\xi$ is the cutting efficiency $\left(\mathrm{mm}^{2} / \mathrm{kJ}\right)$ and $\eta$ is the gas consumption efficiency $\left(\mathrm{g} / \mathrm{mm}^{2}\right), p$ the laser power $(\mathrm{kW}), v$ the cutting speed $(\mathrm{mm} / \mathrm{s}), t$ the material thickness $(\mathrm{mm})$, and $m \cdot$ the mass flow rate $(\mathrm{g} / \mathrm{s})$. Then, the calculated results were compared with those of the reference CN0 nozzle and summarized in Table 5. 
Table 5. Cutting and gas consumption efficiency.

\begin{tabular}{|c|c|c|c|c|c|c|c|c|}
\hline \multirow{2}{*}{$\begin{array}{c}\text { Sample } \\
\text { Thickness }\end{array}$} & \multirow{2}{*}{$\begin{array}{c}\text { Used } \\
\text { Nozzle }\end{array}$} & \multicolumn{2}{|c|}{ Operating Conditions } & \multirow{2}{*}{$\begin{array}{l}\text { Mass Flow } \\
\text { Rates }[\mathrm{g} / \mathrm{s}]\end{array}$} & \multicolumn{2}{|c|}{ Cutting Efficiency $(\xi)$} & \multicolumn{2}{|c|}{$\begin{array}{l}\text { Gas Consumption } \\
\text { Efficiency }(\eta)\end{array}$} \\
\hline & & $\mathbf{P}[$ bar $]$ & $\mathrm{U}[\mathrm{m} / \mathrm{min}]$ & & $\begin{array}{c}(\xi) \\
{\left[\mathrm{mm}^{2} / \mathrm{kJ}\right]}\end{array}$ & $\begin{array}{c}(\xi) \% \\
\text { w.r.t CN0 }\end{array}$ & $\begin{array}{c}(\eta) \\
{\left[\mathrm{g} / \mathrm{mm}^{2}\right]}\end{array}$ & $\begin{array}{c}(\eta) \% \\
\text { w.r.t } \mathrm{CNO}\end{array}$ \\
\hline \multirow{4}{*}{$2 \mathrm{~mm}$} & CNO & 20.00 & 4.5 & 16.84 & 50 & & 0.112 & \\
\hline & SNA & 14.25 & 8.0 & 6.46 & 89 & $+78 \%$ & 0.024 & $+78 \%$ \\
\hline & SNB & 7.50 & 6.0 & 5.42 & 67 & $+33 \%$ & 0.027 & $+76 \%$ \\
\hline & SNC & 4.70 & 4.5 & 4.31 & 50 & - & 0.028 & $+74 \%$ \\
\hline \multirow{4}{*}{$4 \mathrm{~mm}$} & CNO & 20.00 & 2.0 & 16.84 & 44 & & 0.126 & \\
\hline & SNA & 14.25 & 4.0 & 6.46 & 89 & $+100 \%$ & 0.024 & $+81 \%$ \\
\hline & SNB & 7.50 & 3.5 & 5.42 & 78 & $+75 \%$ & 0.023 & $+82 \%$ \\
\hline & SNC & 4.70 & 3.0 & 4.31 & 67 & $+50 \%$ & 0.021 & $+83 \%$ \\
\hline \multirow{3}{*}{$6 \mathrm{~mm}$} & SNA & 14.25 & 2.5 & 6.46 & 83 & - & 0.025 & - \\
\hline & SNB & 7.50 & 2.0 & 5.42 & 67 & - & 0.027 & - \\
\hline & SNC & 4.70 & 2.0 & 4.31 & 67 & - & 0.021 & - \\
\hline
\end{tabular}

Concerning the experimental results, Figures 8-10 and Table 4 demonstrate that the cutting capability was enhanced by using the proposed supersonic nozzles compared to the conical one, since the former made feasible the cutting of the $6 \mathrm{~mm}$ thickness samples.

With reference to the measured roughness for the $2 \mathrm{~mm}$ thickness sample, Table 4 proves that the achieved surface roughness, using the supersonic nozzles, is slightly improved with respect to that obtained by the reference conical nozzle. However, these results were accomplished with a significant reduction in the gas pressure of $29 \%, 63 \%$, and $77 \%$ for nozzles SNA, SNB, and SNC, and an increase in the cutting speed of $78 \%$ and $33 \%$ for nozzles SNA and SNB with respect to CNO.

Even if the roughness level was slightly decreased for the $4 \mathrm{~mm}$ thickness sample with respect to the conical nozzle, similar gains in terms of pressure reduction were achieved, but with a more marked cutting speed increase of $100 \%$ and $75 \%$ for nozzles SNA and SNB, respectively. Although the cutting experiment was successfully carried out using the supersonic nozzle SNC with a cutting speed of $5 \mathrm{~m} / \mathrm{min}, 150 \%$ higher than that of $\mathrm{CN} 0$, in some cases, burrs were generated at the bottom of the cut kerf, as shown in Figure 9.

The cutting experiments on $6 \mathrm{~mm}$ thickness samples using the supersonic nozzles revealed the feasibility of the operation, even if accompanied by a surface roughness quality less than that of the samples of 2 and $4 \mathrm{~mm}$ thickness. However, the surface roughness quality was enhanced by the increase of the operating pressure; the worst cutting quality was achieved with nozzle SNC when operated at the lowest stagnation pressure of 4.7 bar.

Moreover, Table 4 shows that the cutting edge inclination angles remain small and always positive, with no significant differences between the proposed nozzles and the reference conical one.

Table 5 clearly demonstrates that, for the $2 \mathrm{~mm}$ thickness samples, the cutting efficiency was increased up to $78 \%$ and $33 \%$ for SNA and SNB, respectively, and accompanied with a significant increase of the average gas consumption efficiency of up to $76 \%$ compared to that of $\mathrm{CN} 0$. Similar results were achieved for the $4 \mathrm{~mm}$ thickness samples, with an increase of the cutting efficiency up to $100 \%$, $75 \%$, and $50 \%$ for SNA, SNB, and SNC, respectively, and a significant increase of the average gas consumption efficiency of up to $83 \%$ compared to that of nozzle CN0.

\section{Conclusions}

In the present work, a total of three different supersonic nozzles were designed based on the gas dynamics theory and manufactured by means of WEDM. The gas-assisted laser cutting flow through these supersonic nozzles, operated at the desired-design conditions and through a reference conical nozzle, operated at 20 bar, was numerically modeled. Furthermore, the numerically predicted results were compared with the Schlieren experimental measurements. Several cutting experiments were 
conducted on stainless steel samples (AISI 316) of various thicknesses $(2,4$, and $6 \mathrm{~mm}$ ) to comparatively assess the performances of the inert gas-assisted laser cutting based on the use of both nozzle types. The main findings of the work can be summarized as in the following:

- The gas-assisted laser cutting flow through the different supersonic nozzles was efficiently modeled using the proposed model without the occurrence of undulating shock waves in the divergence sections.

- The numerically predicted results are quantitatively in high agreement with the experimental results obtained by Schlieren visualizations in terms of flow structure and mass flow rate, with a peak percentage error of $13.5 \%$.

- A significant reduction of the mass flow rate in the range of $62 \%$ to $74 \%$ was obtained using supersonic nozzles operated at the desired-design condition, in comparison with the conical nozzle. Thus, supersonic nozzles allow the increase of both the energetic cutting efficiency by more than $33 \%$ for $2 \mathrm{~mm}$ thickness samples and more than $50 \%$ for $4 \mathrm{~mm}$ samples, while the gas consumption efficiency is significantly increased in the range of $74 \%$ to $83 \%$.

- The use of supersonic nozzles extends the capability of the process in terms of allowable thickness. For the samples of 2 and $4 \mathrm{~mm}$ thickness, the achieved cutting edge quality is substantially comparable with that of the conical nozzle, but this is accompanied with a noteworthy increase of the cutting speed in the range between $75 \%$ and $100 \%$.

- According to the material and thickness of the samples, the type of the assistance gas and the operating pressure are specified. The achieved numerical and experimental results however suggested the use of nozzles with small divergence angles in order to avoid high flow fluctuations and hence the enlargement of the exit jet width (jet radius).

It can then be concluded that the same cutting quality obtained by the conical nozzle, operated at 20 bar, can be achieved by the proposed supersonic nozzles operated at much lower stagnation pressures (from 5 to 8 bar). This finding can significantly help to increase the cutting speed, to reduce the inert gas consumption rate, and hence reduce the overall operational cost of high-pressure laser cutting.

Author Contributions: Conceptualization, L.O.; methodology, L.O. and M.D.; software, L.O., M.D. and B.R.; validation, L.O., M.D. and B.R.; formal analysis, L.O., M.D. and B.R.; data curation, L.O., M.D. and B.R.

Funding: This research received no external funding.

Acknowledgments: Authors would like to thanks Libor Mrna from Institute of Scientific Instruments-ISI-Brno-Czech Republic for the experimental flow rate measurements and Marco Montani from Optoprim srl for the support in the laser cutting Experiments.

Conflicts of Interest: The authors declare no conflict of interest.

\section{References}

1. Steen, W.M.; Mazumder, J. Laser Material Processing, 4th ed.; Springer: London, UK, 2010; ISBN 978-1-84996-061-8.

2. Dowden, J. The Theory of Laser Materials Processing; Springer: Dordrecht, The Netherlands, 2008.

3. Anderson, J.D. Fundamentals of Aerodynamics, 5th ed.; McGraw-Hill: New York, NY, USA, 2009.

4. Cantwell, B.J. Fundamentals of Compressible Flow, 3rd ed.; Stanford University Press: Stanford, CA, USA, 2007.

5. Man, H.C.; Duan, J.; Yue, T.M. Dynamic characteristics of gas jets from subsonic and supersonic nozzles for high pressure gas laser cutting. Opt. Laser Technol. 1998, 30, 497-509. [CrossRef]

6. Man, H.C.; Duan, J.; Yue, T.M. Analysis of the dynamic characteristics of gas flow inside a laser cut kerf under high cut-assist gas pressure. J. Phys. D Appl. Phys. 1999, 32, 1469-1477. [CrossRef]

7. Boutinguiza, M.; Pou, J.; Lusquiños, F.; Quintero, F.; Soto, R.; Pérez-Amor, M.; Watkins, K.; Steen, W.M. CO 2 laser cutting of slate. Opt. Lasers Eng. 2002, 37, 15-25. [CrossRef]

8. Lin, X.; Wang, P.; Zhang, Y.; Ning, Y.; Zhu, H. Theoretical and experimental aspects of laser cutting using direct diode laser source based on multi-wavelength multiplexing. Opt. Laser Technol. 2019, 114, 66-71. [CrossRef] 
9. Oh, S.Y.; Shin, J.S.; Kim, T.S.; Park, H.; Lee, L.; Chung, C.-M.; Lee, J. Effect of nozzle types on the laser cutting performance for 60-mm-thick stainless steel. Opt. Laser Technol. 2019, 119, 105607. [CrossRef]

10. Shin, J.S.; Oh, S.Y.; Park, H.; Chung, C.-M.; Seon, S.; Kim, T.-S.; Lee, L.; Lee, J. Laser cutting of steel plates up to $100 \mathrm{~mm}$ in thickness with a 6-kW fiber laser for application to dismantling of nuclear facilities. Opt. Lasers Eng. 2018, 100, 98-104. [CrossRef]

11. Seon, S.; Shin, J.S.; Oh, S.Y.; Park, H.; Chung, C.-M.; Kim, T.-S.; Lee, L.; Lee, J. Improvement of cutting performance for thick stainless steel plates by step-like cutting speed increase in high-power fiber laser cutting. Opt. Laser Technol. 2018, 103, 311-317. [CrossRef]

12. Tamura, K.; Ishigami, R.; Yamagishi, R. Laser cutting of thick steel plates and simulated steel components using a $30 \mathrm{~kW}$ fiber laser. J. Nucl. Sci. Technol. 2016, 53, 916-920. [CrossRef]

13. Golnabi, H.; Bahar, M. Investigation of optimum condition in oxygen gas-assisted laser cutting. Opt. Laser Technol. 2009, 41, 454-460. [CrossRef]

14. Oh, S.; Lee, I.; Park, Y.-B.; Ki, H. Investigation of cut quality in fiber laser cutting of CFRP. Opt. Laser Technol. 2019, 113, 129-140. [CrossRef]

15. Quintero, F.; Pou, J.; Fernández, J.L.; Doval, A.F.; Lusquiños, F.; Boutinguiza, M.; Soto, R.; Pérez-Amor, M. Optimization of an off-axis nozzle for assist gas injection in laser fusion cutting. Opt. Lasers Eng. 2006, 44, 1158-1171. [CrossRef]

16. Guo, S.; Jun, H.; Lei, L.; Yao, Z. Numerical analysis of supersonic gas-dynamic characteristic in laser cutting. Opt. Lasers Eng. 2009, 47, 103-110. [CrossRef]

17. Jun, H.; Guo, S.; Lei, L.; Yao, Z. Characteristic analysis of supersonic impinging jet in laser machining. Int. J. Adv. Manuf. Technol. 2008, 39, 716-724. [CrossRef]

18. Chen, K.; Yao, L.; Modi, V. Gas dynamic effects on laser cut quality. J. Manuf. Processes 2001, 3, 38-49. [CrossRef]

19. Chen, K.; Yao, Y.L.; Modi, V. Gas jet-workpiece interactions in laser machining. J. Manuf. Sci. Eng. 2000, 122, 429. [CrossRef]

20. Zhang, C.; Wen, P.; Yuan, Y.; Fan, X. Evaluation and optimal design of supersonic nozzle for laser-assisted oxygen cutting of thick steel sections. Int. J. Adv. Manuf. Technol. 2016, 86, 1243-1251. [CrossRef]

21. Marimuthu, S.; Nath, A.K.; Dey, P.K.; Misra, D.; Bandyopadhyay, D.K.; Chaudhuri, S.P. Design and evaluation of high-pressure nozzle assembly for laser cutting of thick carbon steel. Int. J. Adv. Manuf. Technol. 2017, 92, 15-24. [CrossRef]

22. Darwish, M.; Orazi, L.; Angeli, D. Simulation and analysis of the jet flow patterns from supersonic nozzles of laser cutting using OpenFOAM. Int. J. Adv. Manuf. Technol. 2019, 102, 3229-3242. [CrossRef]

23. Darwish, M.; Mrňa, L.; Orazi, L.; Reggiani, B. Numerical modeling and Schlieren visualization of the gas-assisted laser cutting under various operating stagnation pressures. Int. J. Heat Mass Transfer 2019, 118965. [CrossRef]

24. Andersson, B.; Andersson, R.; Hakansson, L.; Mikael, M.; Sudiyo, R.; Wachem, B.V. Computional Fluid Dynamics for Engineers; Cambridge University Press: New York, NY, USA, 2012.

25. Lomax, H.; Pulliam, T.; Zingg, D. Fundamentals of Computational Fluid Dynamics; Springer-Verlag Berlin and Heidelberg GmbH \& Co. KG: Berlin, Germany, 2001.

26. AMES Research Staff. Equations, Tables, and Charts for Compressible Flow. Available online: https://www.nasa. gov/sites/default/files/734673main_Equations-Tables-Charts-CompressibleFlow-Report-1135.pdf (accessed on 22 September 2019).

27. Tseng, C.-S.; Chen, C.-M.; Wang, C.-C. A visual observation of the air flow pattern for the high speed nozzle applicable to high power laser cutting and welding. Int. Commun. Heat Mass Transfer 2013, 49, 49-54. [CrossRef]

28. Pope, S. Turbulent Flows, 10th ed.; Cambridge University Press: Cambridge, UK, 2013.

29. OpenFOAM Programmer's Guide. Available online: http:/foam.sourceforge.net/docs/Guides-a4/ ProgrammersGuide.pdf (accessed on 24 July 2019).

30. OpenFOAM User Guide. Available online: http://foam.sourceforge.net/docs/Guides-a4/ OpenFOAMUserGuide-A4.pdf (accessed on 13 September 2019).

31. Gnani, F.; Lo, K.; Zare-Behtash, H.; Kontis, K. Shock wave diffraction phenomena around slotted splitters. Aerospace 2015, 2, 1-16. [CrossRef] 
32. Settles, G.S. Schlieren and shadowgraph techniques: Visualizing phenomena in transparent media. In Proceedings of the Schlieren and Shadowgraph Imaging in the Great Outdoors; Springer: New York, NY, USA, 1999.

33. Darwish Ahmad, A.; Abubaker, A.; Salaimeh, A.; Akafuah, N. Schlieren visualization of shaping air during operation of an electrostatic rotary bell sprayer: Impact of shaping air on droplet atomization and transport. Coatings 2018, 8, 279. [CrossRef] 